\title{
A microcomputer-based software package for eye monitoring research
}

\author{
GEORGE W. MCCONKIE \\ University of Illinois at Urbana-Champaign, Urbana, Illinois \\ CHARLES W. SCOUTEN \\ Stoelting Company, Chicago, Illinois \\ and \\ PATRICK K. BRYANT and JERRY WILSON \\ Reading Technology, Inc., Savoy, Illinois
}

\begin{abstract}
A software package is described that collects and reduces eye behavior (eye position and pupil size) data using an IBM-compatible personal computer. Written in the $\mathrm{C}$ language for speed and portability, the package includes several unique features: data can be collected simultaneously from other sources (e.g., EEG, EMG), logically defined events can be detected in real time on any data channel, and either of two types of data matrix can be produced. Data reduction algorithms and data structures are described.
\end{abstract}

The monitoring of eye behavior, including eye position and pupil size, has proven to be useful in studying issues in a number of different fields, including reading (Rayner, 1983), music reading (Sloboda, 1978), X-ray examination (Nodine \& Kundel, 1987), map design (Antes, Chang, \& Mullis, 1985), mathematics education (Suppes, Cohen, Laddaga, Anliker, \& Floyd, 1982), chess (Chase \& Simon, 1973), art and design (Lochegr \& Nodine, 1987), mental load (Kahneman \& Beatty, 1966), emotional response (Hess, 1972), and oculomotor control (Zuber, 1981). Almost any area of research that studies either the acquisition and use of visually presented information or changes in emotional response or arousal can benefit from the recording of eye position or pupil size.

Increased interest in monitoring the eyes has stimulated the development of new devices for monitoring eye behavior. However, a major factor that has prevented a more widespread use of eye monitoring has been the amount of software development required before research is possible. Each laboratory typically writes the software necessary to collect and reduce data with the particular equipment configuration being used.

Recent widespread acceptance of the IBM-initiated standard among personal computers, together with the continually increasing speed of computers of this family, suggests the appropriateness of developing a set of application programs for this computer family that can be used with a variety of eye behavior monitoring devices. The present paper describes a set of programs that was developed spe-

Address correspondence to George W. McConkie, Center for the Study of Reading, University of Illinois at Urbana-Champaign, 51 Gerty Dr., Champaign, IL 61820. cifically for the Stoelting Wide Angle Eye Movement Tracker and TV Pupillometer System, but which is compatible with other eye monitoring equipment.

\section{SOME BASIC CONCEPTS}

The Stoelting tracker/pupillometer, a device that monitors the eyes by means of a video camera, contains hardware that processes each video frame to determine the size and location of the pupil. It samples eye position 60 times per second and gives as output three digital values: 7 bits each for horizontal and vertical eye position and 8 bits for pupil size. In addition, it has a field-of-view camera that allows the stimulus field to be displayed on a monitor, with a fixation spot that moves in response to movements of the eyes. In these respects, the equipment is quite similar to video-camera-based eye monitoring equipment produced by other companies, and is distinguished primarily by its relatively low price.

Each type of eye monitoring device currently available has strengths and weaknesses (Young \& Sheena, 1975). Video-camera-based equipment tends to be relatively easy to use, gives an indication of both horizontal and vertical eye position, typically produces pupil size information as well as eye position, and ordinarily provides output showing the direction of gaze superimposed on a television image of the stimulus field. At the same time, most equipment of this type has a relatively slow sampling rate (i.e., 60 samples per second), accuracy to only about $1^{\circ}$ of visual angle, and a noise level that prevents the reliable detection of small eye movements. Furthermore, there are occasionally artifacts in the signal that result from blinks or failures in the pupil-tracking algorithms that may not reflect actual eye movements or pupil size changes. These 
characteristics make it impossible for equipment of this type to give a precise specification of the fine-grained characteristics of eye movements. For example, with samples taken only every $16 \mathrm{msec}$, it is not possible to specify the precise time at which a 30-msec saccadic eye movement began and ended or to describe its dynamic characteristics. Also, what appears to be a single eye fixation in the data may actually be a series of eye fixations separated by short saccades. However, since most types of research can be carried out without a higher degree of precision, the equipment is quite adequate for many purposes.

In developing software for video-camera-based equipment, it is necessary to deal with the problems of noise and indeterminacy in the data and to provide the researcher with as much information as possible about the eyes' behavior without falsely interpreting noise and artifacts as eye movements. The effects of the choice of values for parameters in the reduction of noisy eye movement data has been vividly described by Karsh and Breitenbach (1983). The goal of the present development was to deal with the problems posed by video-camera-based eye movement data in a way that would maximize the valid information available and, at the same time, would handle appropriately the signal characteristics of other types of eye monitoring devices, including those that sample at other rates and those that have other noise levels and characteristics. The data collection software has the flexibility to collect data from a variety of input ports on the computer, depending on the hardware configuration of the system. The data reduction software has a number of parameters that allow it to be "tuned" to properly handle eye movement data having different characteristics. Finally, diagnostic information is provided in the data matrix to indicate places in the data where noise characteristics are such that the validity of decisions being made is lower than normal. In this way, the more questionable data can be excluded from use in data analysis, if desired.

When eye position is monitored by a tracking device that samples very rapidly (e.g., every millisecond) and with low noise levels, there is seldom a question about whether a saccade occurred; indeterminacy of when it began and ended is within a range of a few milliseconds. Although the software described here can collect and reduce data in which fixations and saccades are always clearly specified, we discuss in this article the more difficult case in which there is greater indeterminacy. In this latter case, it is not appropriate to speak of fixations and saccades, since these cannot be determined precisely. Rather, we speak of a gaze, which can be defined as the period of time between the onset of one stable period in the eye movement data and the next stable period in which the eyes are directed toward a new location. Each gaze, by definition, has an initial stable period. The gaze may also have a period at the end that is not stable but that shows a transition toward the next gaze location. The amount of variability in the data that is still classified as stable and the amount of change in gaze location that is required in order to reliably detect the fact that the eyes have shifted to a new gaze location are controlled by parameters in the program.

\section{THE COMPUTER PROGRAMS}

The eye monitoring software package presently consists of four programs: one for data collection, two for data reduction, and one that allows the user to modify calibration values in the data file. They run on IBMcompatible personal computers with $256 \mathrm{~K}$ of memory and a hard disk. The programs are written primarily in the Microsoft $C$ language, using the Microsoft $C$ compiler to achieve both speed and portability. However, this language choice presented certain problems that are discussed below. Flexibility is achieved through parameter files that specify a number of the programs' internal characteristics.

\section{Data Collection Program}

The Data Collection Program is designed to collect eye position and pupil size data, as well as data of other kinds, including buttonpresses and analog data. Up to eight 16bit input ports can be recorded. The rate of sampling is determined externally. The program samples on the leading edge of a positive-going pulse that is generated by the eye monitoring device or produced by a clock. The program also allows the user to display stimulus patterns on the computer screen, advancing pages with keypresses or buttonpresses, or to use external displays, perhaps projecting slides or physically placing photographs in the field of view.

The user runs the Data Collection Program by typing its name plus the names of three files: a Parameter File, a Display Control File, and a Data File. The Parameter File and Display Control File are ASCII files that can be prepared and modified using any text editor capable of producing pure ASCII output. One additional file that contains the hardware initialization code is required. This file is used to establish communication between the computer and the eye monitoring equipment. For an interface, we currently use the Qua Tech PXB-721 Parallel Expansion Board, for which A/D modules are also available for monitoring analog signals. If different equipment is used, then this file can be altered to reflect the characteristics of the equipment.

Upon being run, the Data Collection Program requests the subject's name and identification number and an experimental condition number. All are necessary parameters, but shell scripts (i.e., DOS batch files) can be written that automatically specify some of these parameters. This procedure reduces the likelihood of experimeter error.

The parameters that are contained in the Parameter File are listed in Table 1. These parameters define the hardware environment, the channels on which data are to be collected, and information to be used in the calibration 
Table 1

Data Collection Program Parameters: Parameter File

Number of data collection channels defined.

For each channel (0-7):

Channel title

Address (I/O Port)

Data width (8 or 16 bits)

Read on interrupt $(1=$ enabled; $0=$ disabled $)$

Interrupt request line that is active.

Type of display (Internal or External, where Internal is a display on the computer monitor).

Number of calibration locations.

For each calibration location:

$X$ value in display space

$Y$ value in display space

$X$ location on computer screen

$Y$ location on computer screen

Calibration threshold on $X$ data.

Calibration threshold on $Y$ data.

Display colors:

Text color

Background color

Emphasized text color

Type of subject interaction during calibration (Keyboard or Event).

Type of subject interaction during data collection (Keyboard or Event).

process. This file is read and the parameters are invoked as part of the initialization process each time the program is run.

The Display Control File defines the sequence of events that are to occur in the experiment. By means of a series of dot commands, shown in Table 2, displays can be put onto the screen, data collection channels can be enabled and disabled, and calibrations can be taken as desired. Alphanumeric display pages can be included in the Display Control File and can use the IBM extended character set, thus giving some limited graphics capability.

The Data Collection Program contains a calibration procedure, which, when called in the internal display mode (i.e., when using the computer screen to display stimulus material), displays a grid of Xs on the computer screen at locations defined in the Parameter File. The color of each $\mathrm{X}$ is modified, in sequence, as a signal for the subject to fixate that point and press a key or button. When the key is pressed, the computer samples the eye data values, saving the values in a calibration table. This is repeated for each location, and the values obtained are compared with the prior values. If eye position values

Table 2

Data Collection Program: Dot Commands in Display Control File .ep-end of page.

.cb-calibrate.

nc-no collect (disable all data collection).

.cd-collect data (enable data collection).

.ac-activate a channel for data collection.

da-deactivate a channel so data not collected.

.ed-event definition.

eu-event undefined. deviate by more than the $X$ or $Y$ calibration threshold values specified in the parameter file, additional samples are taken from that location. This sampling continues until two successive samples taken from each location fall within the threshold values specified or until the experimenter manually exits the calibration procedure. This calibration procedure can be repeated at any time during the data collection process.

When the Data Collection Program is initially called, a nine-point matrix of digits appears on the screen while the experimenter adjusts the eye monitoring equipment. During this time, the values of data channels that are defined as eye position and pupil size are displayed continuously on the bottom of the computer screen. Also during this time, the computer identifies the range of values obtained from the pupil size channel, if available. Thresholds then are calculated for later use in identifying blinks. A keyboard command next advances the program to the actual data collection procedures, where calibrations and data collection can occur.

In the Data File that is produced by the Data Collection Program, the first 2048 bytes are used to store complete information about the data collection session (see Table 3), including data from each occurrence of the calibration task. Following this, a data record of variable length is created to contain the data collected during each display. This record indicates the channels on which data were collected during that display, an experimental condition identifier for that display, and the actual data, which consists of a 2-byte integer per sample for each channel.

One additional feature of the Data Collection Program is the ability to detect events and to take action when they occur. An event is the occurrence of a logical condition in the data coming from a specified data channel. An event is defined by an identifying title, a channel number, a threshold value, and a logical condition, including equal, not equal, greater than, less than, bitwise AND, and bitwise OR. Each defined event is tested as each data sample is taken. If an event tests as "true," then immediate action can be taken. Up to four events can be specified. At present, this feature is used to respond to the press of buttons on one of the data channels, which allows the system to advance the display or to simply record a subject's response. However, this feature provides the basis for a large number of potential response-contingent stimulus manipulations, especially since the events can be defined on channels other than the eye behavior channels. More detail concerning the techniques used to allow a $C$ language program to deal with real-time events is presented below.

\section{Data Reduction Programs}

Two data reduction programs have been developed. One program is for situations in which the primary interest is in eye position data that are of a fixation-saccade type, and the other is for situations in which the interest is in nonsaccade data, such as pursuit eye movements, pupil 
Table 3

Data Collection Program: Records in the Data File

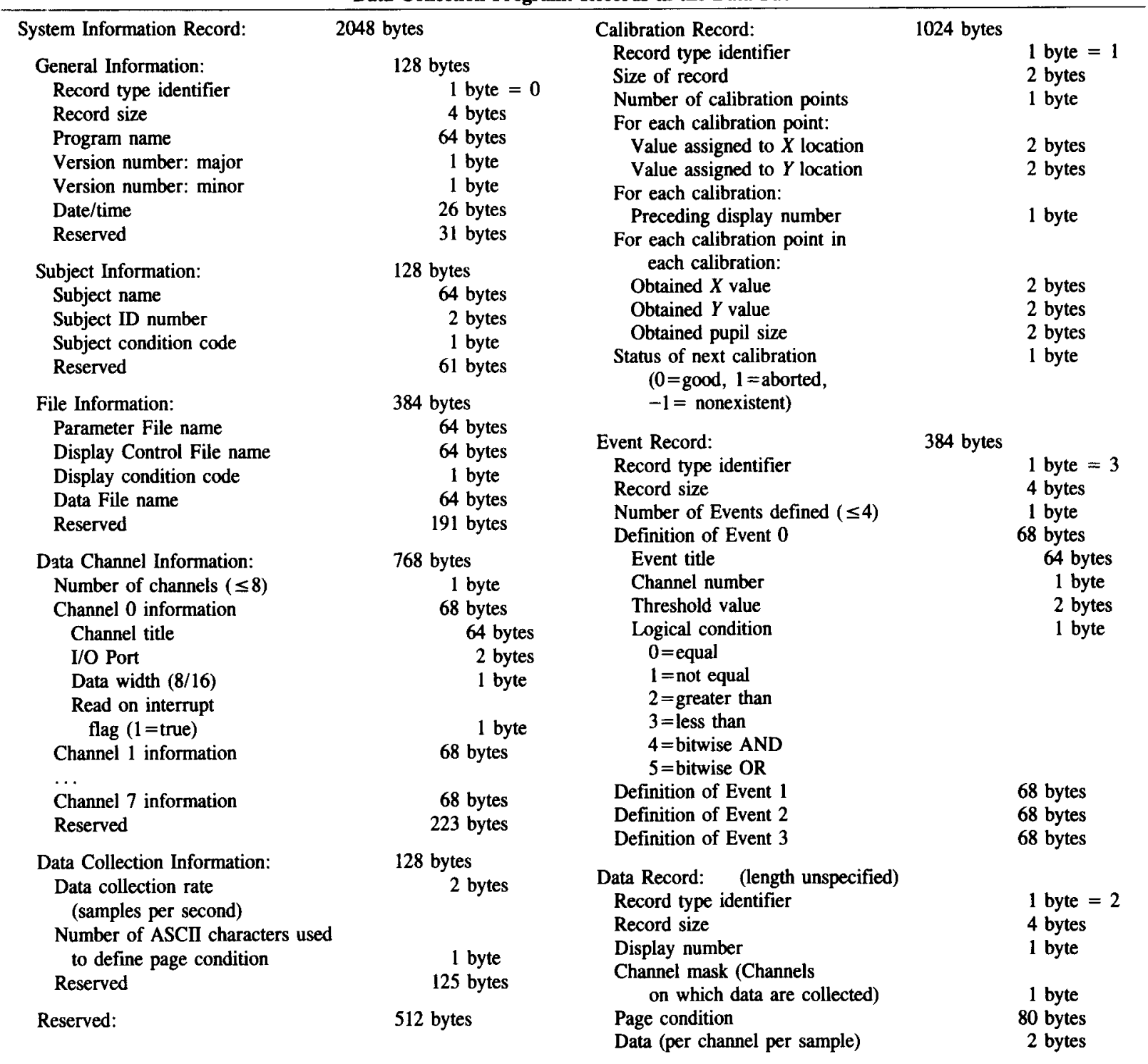

size, or EEG. The first program, the Gaze-Oriented Reduction Program, produces a matrix in which each data row corresponds to a gaze or fixation-saccade pair. The second program, the Stream Data Reduction Program, is for use when the data cannot appropriately be partitioned into gaze segments. This program produces a row in the data matrix for every data sample taken. However, if eye position data were collected, then the matrix can include columns indicating eye position, the state of the eyes (i.e., stable or in transition), and the beginning and ending times of gazes.

Gaze-Oriented Data Reduction Program. Table 4 contains definitions and parameters used in the GazeOriented Data Reduction Program, and Table 5 presents the general algorithm used in identifying gazes. The algorithm identifies periods of stability in the data, then examines the periods of instability lying between them, attempting to resolve them as either periods of noise in the data or transition periods (i.e., eye movements taking the eyes to a new location). If the period of instability has characteristics that make it inappropriate to interpret in either of these simple ways, then a complex transition analysis is carried out that attempts to break the period into additional gazes using recursion with new parameters. The program produces an ASCII data matrix that contains a row of data for each identified gaze. The columns in the matrix are listed in Table 6 . The matrix is in a form typically used with statistical programs and contains information about the location and duration of each gaze, the duration of the stable part of the gaze, and indications of data quality, such as the percentage of samples with nonaberrant values, amount of drift during the stable part of a gaze (a possible indicator of small, undetected saccades), and abnormal pupil size (an indicator of a blink).

Once a gaze has been identified, averages of the stable $X$ and $Y$ channel values are calculated. These averages 
Table 4

Gaze-Oriented Data Reduction Program:

Definitions and Parameters

\begin{tabular}{ll}
\hline Term or Parameter* & \multicolumn{1}{c}{ Definition } \\
\hline$X$ Channel & $\begin{array}{l}\text { The data channel that gives information about } \\
\text { horizontal eye position. } \\
\text { The data channel that gives information about } \\
\text { vertical eye position. } \\
\text { Gaze }\end{array}$ \\
$\begin{array}{l}\text { The eye movement record is used to segment } \\
\text { time into discrete periods, each termed a Gaze. } \\
\text { Each Gaze begins when the eyes stabilize in a } \\
\text { new position. }\end{array}$
\end{tabular}

Stable Period

The initial period of a Gaze, during which the Term or Parameter* Definition

Reduced Local Gaze A specified number of samples, fewer than the Local Gaze Duration, that meet the Local Gaze Threshold Definition for a Local Gaze. The Reduced Local Gaze is used in Complex Transition Analysis.

Reduced Local The number of samples in a Reduced Local Gaze Duration Gaze. (3)

Aberrant Value A data value from either the $X$ or $Y$ channel that differs from both the preceding and following data values by at least a specified amount, and in the same direction. Thus, it is a local peak or valley in the data stream, and is assumed to represent noise rather than actual change in eye position.

Gaze Duration The number of samples included in a Stable Period.

Transition Period The final period of a Gaze, during which the

Transition Length The distance between the Gaze Locations that

Transition Direction The angle of the line connecting two successive

Transition Duration The number of samples included in a Transi-

Local Gaze A specified number of samples in which the

Lacal Gaze Duration The number of samples in a Local Gaze. (5)

Local Gaze

Threshold $\mathrm{X}$

Local Gaze

Threshold $Y$

Transition Data

Consistent Transition Data Inconsistent

Transition Data eyes move to a new location. precede and follow the Transition Period, measured in units appropriate to the stimulus display. Gaze Locations, measured in degrees, where zero deg is a horizontal line extending directly rightward from a Gaze Location. Locations above that location have positive values, and locations below have negative values. tion Period. maximum and minimum values on both the $X$ and the $Y$ channels differ by no more than the values defined as thresholds for those channels.

The maximum difference that can exist among a set of $X$ data values and still be considered a Local Gaze. (1)

The maximum difference that can exist among a set of $Y$ data values and still be considered a Local Gaze. (1)

A string of data values that does not contain a region that satisfies the definition of a Local Gaze. Thus, a candidate for containing a Transition Period. Transition Data values from one channel that change in only one direction.

Aberrant Value

Threshold

Transition Analysis

The threshold that defines an aberrant data value. (2)

A procedure for examining Transition Data in order to determine whether or not a Transition Period exists.

Minimum Gaze Duration

Successive Gaze Thresholds$X \& Y$

Complex Transition Period

Transition Duration Threshold

Complex Transition Analysis

Transition Data values from one channel that both increase and decrease.
The minimum number of samples that can exist between the initial samples of two successive Local Gazes, without collapsing these into a single Gaze. Notice that this parameter is used in dealing with the case of overlapping Local Gazes. (3)

If the locations of two successive Gazes differ by less than the Successive Gaze Thresholds on both $X$ and $Y$ channels, they are collapsed into a single Gaze. (1)

Transition Data that (a) contain more samples than specified by Transition Duration Threshold, or (b) have fewer samples than this threshold, are inconsistent (see definition above), and with preceding and following Gaze Locations that differ by more than the Successive Gaze Threshold. Transition Data not meeting either criterion are dealt with in the normal Transition Analysis.

A threshold used in determining whether a set of Transition Data constitutes a simple or complex transition period. (3)

A procedure for further analyzing a Complex Transition Period to determine whether it can be broken up into additional Gazes. This procedure uses the Reduced Local Gaze in its attempt to find Stable Periods.

Note-Default values for parameters used with Stoelting Pupillometer are given in parentheses.

*Parameters are typed in italic.

are used to determine where the eyes were directed in the stimulus display during that gaze. The algorithm used in this mapping process produces a solution similar to that of the linear interpolation procedure suggested by McConkie, Zola, Wolverton, and Burns (1978), but in fact accomplishes this goal using recursion.

The mapping algorithm first divides the stimulus space into quadrangles, based on the locations of the grid of target locations used in the calibration task. The algorithm assigns to each calibration target location the $X$ and $Y$ chan- nel values obtained during the last calibration prior to the display, or the values obtained during the first calibration following it, or an average of the values obtained on these two calibrations. The algorithm then takes the average location of the eyes during the stable part of the first gaze and identifies the quadrangle within which it lies. Two lines are then identified that bisect opposing sides of the quadrangle and that divide it into four smaller quadrangles. Again, the algorithm identifies which of these quadrangles contains the gaze location. This process con- 
Table 5

Gaze-Oriented Data Reduction Program: Procedure

Given: A Local Gaze has been identified.

A. Find the next series of data values that qualifies as a Local Gaze and carry out Transition Analysis.

1. If the new Local Gaze begins one data value after the beginning of the prior Local Gaze, extend the current Gaze to include new data point. Go to $\mathrm{A}$.

2. If the new Local Gaze begins no more than a MINIMUM GAZE DURATION of values following the beginning of the prior Local Gaze, extend the current Gaze to include these data. Go to A. (Note: This includes cases where Local Gazes overlap substantially but are not sequential, indicating the possibility of some movement during the gaze, but too little to identify as saccades.)

3. Else, carry out Transition Analysis to examine the Transition Data.

a. If the difference between either the average $X$ or $Y$ data values from the preceding and following stable periods exceeds the GAZE MOVEMENT THRESHOLD, then set the Movement Flag = TRUE, else set it FALSE. (Set a flag if the eyes have changed location.)

b. Set the Transition Duration Code to indicate the duration of the Transition Period. If Transition Duration (TD) $=0$, then set Transition Duration Code (TDC) $=0$; if $T D=1$, then set TDC $=1$; if TD is greater than 1 but less than the Transition Duration Threshold, then set TDC $=2$; if TD is greater than or equal to the Transition Duration Threshold, then set $\mathrm{TD}=3$.

c. If the Transition Data from either the $X$ or the $Y$ channel are Inconsistent, then set the Consistency Flag = FALSE, else set it TRUE. (Use a flag to indicate whether or not the data show the eyes proceeding consistently in one direction.)

d. If the Movement Flag = TRUE and the Consistency Flag = TRUE and the Transition Duration Code $<3$, then classify this as a clean transition, close out the old Gaze, and go to A. (A short, consistent transition that takes the eyes to a new location are accepted as a saccadic movement.)

e. If the Transition Duration Code $=3$ or if the Movement Flag $=$ TRUE and the Transition Duration Code $=2$ and the Con- sistency Flag = FALSE, then go to 4 and carry out Complex Transition Analysis. (If this is a long transition or if the direction of movement is not consistent, it needs additional analysis.)

f. If the Transition Duration Code $<3$ and the Movement Flag $=$ FALSE and the Consistency Flag = FALSE, then mark all Aberrant Values, combine the preceding and following Stable Periods into one Gaze, and go to $A$. (If this is a period of inconsistent movement with the eyes ending up in the same place, conclude that it is simply a period of noise and include it as part of the gaze.)

g. If the Transition Duration Code $<3$ and the Movement Flag $=$ FALSE and the Consistency Flag = TRUE, then combine the preceding and following Stable Periods into one Gaze, and go to A. (This is a condition in which there is a period of consistent movement that leaves the eyes in the same location. It is included for completeness, but should not normally occur.)

h. If the Transition Duration Code $<3$ and the Movement Flag $=$ TRUE and the Consistency Flag = FALSE, then mark the Aberrant Values. If an Aberrant Value is adjacent to a Stable Period, then join it to that Period, removing it from the Transition Period. The remaining Transition Period is accepted as a legitimate transition. Go to $A$. (If the eyes have moved, but the transition period is noisy, extend the Stable Period if possible and accept the transition.)

B. Carry out Complex Transition Analysis.

1. Mark all Aberrant Values in Transition Data. Consider them to be equal to their bounding values, and reapply Transition Analysis. If this eliminates all Transition Data, go to A. (The transition was apparently simply an extended noise period.)

2. Else use Reduced Local Gaze definition and reapply Transition Analysis to remaining Transition Data. Indicate in the Problem Flag when data show unusual characteristics. Go to A. (This call will find shorter stable periods or periods during which there is more drift or movement, but which do not show the characteristics of saccadic movements. Thus, it has the capability of decomposing a long string of Transition Data into additional gazes.) tinues until the gaze location is within a specified distance of one of the corners of a quadrangle, at which point that corner is taken as the gaze location. Since the algorithm knows the corresponding location of that corner in the stimulus field, the mapping of the data point onto a stimulus location is complete and the stimulus-based gaze location is entered into the matrix. The distance threshold used in this process is a parameter in the program. The gaze location, together with the distance and direction of this gaze from prior and following gazes, are entered into the data matrix.

Special provisions are made in the program to handle a gaze that lies outside the region in which calibration targets were used. This is done by extending the nearest quadrangle to include the data point, estimating $X$ and $Y$ values corresponding to the new corners, and then using the normal mapping algorithm to estimate the location of the gaze on the stimulus.

If an event occurred during a gaze, the matrix also contains information about which event occurred and the time of its occurrence. This can be used to record response times and/or response selection if each event defined is the press of a particular button.

The Gaze-Oriented Data Reduction Program also puts summary information concerning data from non-eyeposition channels into the data matrix, including mean value on that channel during the eye fixation, as well as an indication of the direction and amount of change during the gaze period. Finally, the sample number of the first data sample in the gaze is included. This number provides a pointer from the gaze-based matrix into the original data matrix. Thus, it is possible to write programs that select the data collected on some non-eye-movement channel, starting with the onset of a particular gaze or event.

Stream Data Reduction Program. When the researcher's interest is primarily in changes in pupil size or some other continuous data, such as EEG or smooth pursuit eye movement data, the data matrix for analysis is prepared using the Stream Data Reduction Program. This program creates an ASCII matrix having one row for each sample taken. Subject and condition columns are 
Table 6

Gaze-Oriented Data Reduction Program: Data Matrix

\begin{tabular}{|c|c|c|}
\hline Col. & Bytes & Contents \\
\hline 1. & 4 & Subject number \\
\hline 2. & 2 & Subject condition code \\
\hline 3. & 3 & Display number \\
\hline 4. & As defined & Display condition code \\
\hline 5. & 3 & Gaze number on display \\
\hline 6. & 6 & Eye position on display: $X$ \\
\hline 7. & 6 & Eye position on display: $Y$ \\
\hline 8. & 3 & $\begin{array}{l}\text { Percent of values that are nonaberrant: } \\
X\end{array}$ \\
\hline 9. & 3 & $\begin{array}{l}\text { Percent of values that are nonaberrant: } \\
Y\end{array}$ \\
\hline 10. & 5 & Distance from prior Gaze \\
\hline 11. & 6 & Direction from prior Gaze (degrees) \\
\hline 12. & 5 & Distance to following Gaze \\
\hline 13. & 6 & Direction to following Gaze (degrees) \\
\hline 14. & 4 & Total number of samples in Gaze \\
\hline 15. & 4 & $\begin{array}{l}\text { Number of samples in stable part of } \\
\text { Gaze }\end{array}$ \\
\hline 16. & 7 & Problem Flag \\
\hline 17. & 5 & Sample number of first sample in Gaze \\
\hline 18. & 4 & Number of aberrant samples \\
\hline 19. & 5 & Average $X$ value in Gaze \\
\hline 20. & 5 & Average $Y$ value in Gaze \\
\hline 21. & 4 & Change in $X$ during Gaze \\
\hline 22. & 4 & Change in $Y$ during Gaze \\
\hline
\end{tabular}

For each Event, when it occurs:

$\begin{array}{lll}\text { E1. } & 2 & \text { Event number } \\ \text { E2. } & 4 & \text { Time since beginning of Gaze } \\ \text { E3. } & 5 & \text { Time since beginning of Display }\end{array}$

For each Data Channel other than $X$ and $Y$ Eye Position:

\begin{tabular}{lll} 
D1. & 5 & Mean data value during Gaze \\
D2. & 4 & Maximum data value during Gaze \\
D3. & 4 & Minimum data value during Gaze \\
D4. & 4 & Mode data value during Gaze \\
D5. & 5 & Slope of data during Gaze \\
\hline
\end{tabular}

added to the data. If eye position has also been recorded, then columns can be created that indicate for each sample whether it occurred during a period of stability or transition, how many samples have been taken since the beginning of the gaze, where the eyes were directed during that gaze, and whether there was any problem in the eye position data at that time (e.g., blinks or aberrant values). The occurrence of events can also be noted. With this matrix it is easy to identify the data taken on one channel beginning at the onset of a fixation at a particular location in the display, or beginning at the point where the subject pressed a button or the pupil size exceeded a particular value. Columns in this matrix are listed in Table 7.

\section{Calibration Data Editor}

A fourth program included in the software package allows the experimenter to examine the calibration values contained in a data file. Furthermore, if it is found that a value is incorrect, then the program allows the experimenter to replace the original value with a new one. This is occasionally necessary when a value obtained in one calibration is very deviant from those obtained during other calibrations in the same session.

\section{USING THE C LANGUAGE FOR REAL-TIME PROGRAMMING}

The C Language that is available on the IBM PC was not designed for use in real-time data collection. In particular, it does not provide a method of responding to most interrupts. Attempting to use it to respond directly to interrupts can actually interfere with the proper execution of some of its standard commands. This fact created certain problems in developing the Data Collection Program, particularly the need for interrupt-driven data collection and the need to respond quickly to the occurrence of an event. This problem was dealt with by developing an input channel handler, written in assembly language. This routine receives commands from the $\mathrm{C}$ language program and, in turn, makes information available to it that can be read upon request.

The input channel handler receives instructions from the calling program concerning which channels to take data from, whether to read 8 or 16 bits of data, which port addresses to use, where the data are found, which interrupt request line to respond to, and what the definitions of events are. When activated, the handler then responds to each interrupt by sampling the data from each specified input port and storing it in a buffer. Furthermore, as each sample is taken, that sample is tested against any event definition specified for that channel. If the test succeeds, then a bit is set in a status word to indicate that the event occurred.

The $C$ language program can read the 16-bit status word and a 16-bit error word generated by the input channel

Table 7

Stream Data Reduction Program: Data Matrix

\begin{tabular}{|c|c|c|}
\hline Col. & Bytes & Contents \\
\hline 1. & 4 & Subject number \\
\hline 2. & 2 & Subject condition code \\
\hline 3. & 3 & Display number \\
\hline 4. & As defined & Display condition code \\
\hline 5. & 5 & Data sample number \\
\hline \multicolumn{3}{|r|}{ For Each Channel $(0-7)$ : } \\
\hline \multirow[t]{2}{*}{ Cl. } & 5 & Channel data value \\
\hline & & Gaze Information: \\
\hline G1. & 3 & Gaze number on display \\
\hline G2. & 6 & Eye position on display: $X$ \\
\hline G3. & 6 & Eye position on display: $Y$ \\
\hline G4. & 4 & Total number of samples in Gaze \\
\hline G5. & 4 & Number of samples in stable part of Gaze \\
\hline G6. & 4 & Sample number in the Gaze \\
\hline G7. & 7 & Problem flag for gaze \\
\hline G8. & 4 & Percent of samples that are aberrant \\
\hline G9. & 3 & Blink flag \\
\hline G10. & 2 & Aberrant value flag: $X$ \\
\hline \multirow[t]{2}{*}{ G11. } & 2 & Aberrant value flag: $Y$ \\
\hline & \multicolumn{2}{|c|}{ For Each Defined Event (0-3): } \\
\hline E1. & 2 & Event type \\
\hline E2. & 4 & Number of samples since Gaze beginning \\
\hline E3. & 5 & $\begin{array}{l}\text { Number of samples since display be- } \\
\text { ginning }\end{array}$ \\
\hline
\end{tabular}


handler and can request values from the data buffer. For simply obtaining data, the program can request data from the handler at its own convenience, so long as this occurs frequently enough to avoid overrunning the buffer. However, to respond quickly to events, it is necessary for the $C$ language program to check the status flag frequently to see if an event has occurred. Because the program was written so that it checks this status at least every millisecond, it can respond to an event within $1 \mathrm{msec}$ of the event's occurrence.

\section{CONCLUSION}

Monitoring eye behavior can play any of three different roles in research. First, it provides data for studying oculomotor control, perception, cognition, emotional response, or arousal. Second, eye behavior data can serve as a basis for analyzing simultaneously collected data, thus allowing selection, elimination, or grouping of those data. Third, the eyes can be used to control aspects of the stimulus; for instance, the display can be changed when the eyes are directed at a certain location or a tone can be sounded when the size of the pupil exceeds a certain value.

The software package described above makes it possible to use an IBM-compatible personal computer to acquire and reduce eye behavior data for any of the three purposes just described. Although developed specifically for the Stoelting Wide Angle Eye Movement Tracker and TV Pupillometer System, the package was designed to be compatible with a variety of different eye monitoring devices that sample at different rates and have different noise characteristics. The availability of this software should substantially reduce the time and cost involved in establishing the facilities to obtain eye behavior data. One unique contribution of the software is the ability to simultaneously collect data of other types, such as other physiological measures, buttonpresses, or indications of changes taking place in the stimulus field. The second contribution is a general facility for detecting logically defined events in the data in real time. This capability can be used for making response-contingent stimulus changes, although this currently requires making user-specific modifications to the existing software. These two characteristics open the door to new and innovative roles for eye monitoring in psychological research.

\section{REFERENCES}

Antes, J. R., Chang, K., \& Mullis, C. (1985). The visual effect of map design: An eye-movement analysis. The American Cartographer, $12,143-155$.

Chase, W. G., \& Simon, H. A. (1973). The mind's eye in chess. In W. G. Chase (Ed.), Visual information processing (pp. 215-281). New York: Academic Press.

HEss, E. H. (1972). Pupillometrics: A method of studying mental, emotional, and sensory processes. In N. S. Greenfield \& R. A. Sternbach (Eds.), Handbook of psychophysiology (pp. 491-531). New York: Holt, Rinehart \& Winston.

Kahneman, D., \& Beatty, J. (1966). Pupil diameter and load on memory. Science, 154, 1583-1585.

KARSH, R., \& BreitenBaCh, F. W. (1983). Looking at looking: The amorphous fixation measure. In R. Groner, C. Menz, D. F. Fisher, \& R. A. Monty (Eds.), Eye movements and psychological functions: International views (pp. 53-64). Hillsdale, NJ: Erlbaum.

LOCHEGR, P., \& NoDINE, C. (1987). Symmetry catches the eye. In J. K. O'Regan \& A. Levy-Schoen (Eds.), Eye movements: From physiology to cognition (pp. 353-361). Amsterdam: North Holland.

McConkie, G. W., Zola, D., Wolverton, G. S., \& Burns, D. D. (1978). Eye movement contingent display control in studying reading. Behavior Research Methods \& Instrumentation, 10, 154-166.

Nodine, C. F., \&undel, H. L. (1987). The cognitive side of visual search in radiology. In J. K. O'Regan \& A. Levy-Schoen (Eds.), Eye movements: From physiology to cognition (pp. 578-583). Amsterdam: North Holland.

RAYNER, K. (Ed.). (1983). Eye movements in reading: Perceptual and language processes. New York: Academic Press.

SLoBoda, J. A. (1978). The psychology of music reading. Psychology of Music, 6, 3-20.

Suppes, P., Cohen, M., Laddaga, R., Anliker, J., \& Floyd, R. (1982). Research on eye movements in arithmetic performance. In R. Groner \& P. Fraisse (Eds.), Cognition and eye movements (pp. 5773). Amsterdam: North Holland.

YounG, L. R., \& SHEENA, D. (1975). Survey of eye movement recording techniques. Behavior Research Methods \& Instrumentation, 7, $397-429$.

ZuBER, B. L. (Ed.). (1981). Models of oculomotor behavior and control. Boca Raton, FL: CRC Press. 